Supporting Information for

\title{
Electronic Ground State of Higher Acenes
}

\author{
De-en Jiang ${ }^{1, *}$ and Sheng Dai ${ }^{1,2}$ \\ ${ }^{1}$ Chemical Sciences Division and ${ }^{2}$ Center for Nanophase Materials Sciences, Oak Ridge \\ National Laboratory, Oak Ridge, Tennessee 37831
}

*To whom correspondence should be addressed. E-mail: jiangd@ornl.gov. Phone: (865)574-5199. Fax: (865) 576-5235. 
Table S-1. Energies (in eV) for different states of acenes

\begin{tabular}{|c|c|c|c|}
\hline n-acene & $\mathrm{NM}^{a}$ & $\mathrm{FM}^{b}$ & $\mathrm{AFM}^{c}$ \\
\hline 5 & -249.554 & -248.842 & -249.554 \\
\hline 6 & -292.851 & -292.435 & -292.851 \\
\hline 7 & -336.143 & -335.939 & -336.151 \\
\hline 8 & -379.434 & -379.398 & -379.502 \\
\hline 9 & -422.723 & -422.81 & -422.882 \\
\hline 10 & -466.017 & -466.198 & -466.267 \\
\hline 11 & -509.472 & -509.562 & -509.651 \\
\hline 12 & -552.858 & -552.911 & -553.026 \\
\hline 13 & -596.276 & -596.244 & -596.399 \\
\hline 14 & -639.664 & -639.581 & -639.768 \\
\hline 15 & -683.031 & -682.93 & -683.128 \\
\hline 16 & -726.378 & -726.296 & -726.486 \\
\hline 20 & -899.654 & -899.769 & -899.968 \\
\hline 40 & -1766.638 & -1766.571 & -1767.005 \\
\hline
\end{tabular}

${ }^{\text {a }} \mathrm{NM}$ refers to the nonmagnetic state, i.e., closed-shell singlet;

${ }^{\mathrm{b}}$ FM refers to the triplet;

${ }^{\mathrm{c}}$ AFM refers to the open-shell singlet. 
Coordinates (in XYZ format) of acenes in their ground state

\begin{tabular}{|c|c|c|c|}
\hline \multicolumn{4}{|c|}{ [5] -acene } \\
\hline \multicolumn{4}{|c|}{6.089494} \\
\hline C & 4.626891 & 6.089893 & 5.000000 \\
\hline C & 14.513114 & 6.089893 & 5.000000 \\
\hline $\mathrm{C}$ & 7.101902 & 6.090336 & 5.000000 \\
\hline C & 12.038098 & 6.090336 & 5.000000 \\
\hline C & 8.341850 & 6.770103 & 5.000000 \\
\hline $\mathrm{C}$ & 10.798152 & 6.770103 & 5.000000 \\
\hline c & 5.886960 & 6.771971 & 5.000000 \\
\hline $\mathrm{C}$ & 13.253040 & 6.771971 & 5.000000 \\
\hline C & 3.445648 & 6.784945 & 5.000000 \\
\hline $\mathrm{C}$ & 15.694354 & 6.784945 & 5.000000 \\
\hline C & 3.445648 & 8.215055 & 5.000000 \\
\hline C & 15.694354 & 8.215055 & 5.000000 \\
\hline C & 5.886960 & 8.228029 & 5.000000 \\
\hline$C$ & 13.253040 & 8.228029 & 5.000000 \\
\hline C & 8.341850 & 8.229897 & 5.000000 \\
\hline C & 10.798152 & 8.229897 & 5.000000 \\
\hline C & 7.101902 & 8.909664 & 5.000000 \\
\hline C & 12.038098 & 8.909664 & 5.000000 \\
\hline C & 4.626891 & 8.910107 & 5.000000 \\
\hline $\mathrm{C}$ & 14.513114 & 8.910107 & 5.000000 \\
\hline C & 9.570000 & 8.910506 & 5.000000 \\
\hline $\mathrm{H}$ & 2.495256 & 8.751116 & 5.000000 \\
\hline $\mathrm{H}$ & 2.495256 & 6.248884 & 5.000000 \\
\hline $\mathrm{H}$ & 4.628549 & 10.002495 & 5.000000 \\
\hline $\mathrm{H}$ & 4.628549 & 4.997505 & 5.000000 \\
\hline $\mathrm{H}$ & 7.101146 & 10.003049 & 5.000000 \\
\hline $\mathrm{H}$ & 7.101146 & 4.996951 & 5.000000 \\
\hline $\mathrm{H}$ & 9.570000 & 10.003527 & 5.000000 \\
\hline $\mathrm{H}$ & 9.570000 & 4.996473 & 5.000000 \\
\hline $\mathrm{H}$ & 12.038856 & 10.003049 & 5.000000 \\
\hline $\mathrm{H}$ & 12.038856 & 4.996951 & 5.000000 \\
\hline $\mathrm{H}$ & 14.511453 & 10.002495 & 5.000000 \\
\hline $\mathrm{H}$ & 14.511453 & 4.997505 & 5.000000 \\
\hline $\mathrm{H}$ & 16.644746 & 8.751116 & 5.000000 \\
\hline $\mathrm{H}$ & 16.644746 & 6.248884 & 5.000000 \\
\hline \multicolumn{4}{|c|}{ [6]-acene } \\
\hline C & 3.975507 & 8.215200 & 5.000000 \\
\hline C & 3.975507 & 6.784800 & 5.000000 \\
\hline C & 5.156201 & 8.910532 & 5.000000 \\
\hline C & 5.156201 & 6.089468 & 5.000000 \\
\hline C & 6.417110 & 8.229031 & 5.000000 \\
\hline C & 6.417110 & 6.770969 & 5.000000 \\
\hline $\mathrm{C}$ & 7.630693 & 8.910456 & 5.000000 \\
\hline C & 7.630693 & 6.089544 & 5.000000 \\
\hline C & 8.872504 & 8.231178 & 5.000000 \\
\hline C & 8.872504 & 6.768822 & 5.000000 \\
\hline C & 10.097581 & 8.912012 & 5.000000 \\
\hline $\mathrm{C}$ & 10.097581 & 6.087988 & 5.000000 \\
\hline C & 11.330000 & 8.231693 & 5.000000 \\
\hline $\mathrm{C}$ & 11.330000 & 6.768307 & 5.000000 \\
\hline $\mathrm{C}$ & 12.562422 & 8.912012 & 5.000000 \\
\hline C & 12.562422 & 6.087988 & 5.000000 \\
\hline & & & \\
\hline
\end{tabular}




\begin{tabular}{|c|c|c|c|}
\hline C & 13.787496 & 6.768822 & 5.000000 \\
\hline & 15.029310 & 8.910456 & 5.000000 \\
\hline & 15.029310 & 6.089544 & 5.000000 \\
\hline C & 16.242893 & 8.229031 & 5.000000 \\
\hline C & 16.242893 & 6.770969 & 5.000000 \\
\hline C & 17.503802 & 8.910532 & 5.000000 \\
\hline C & 17.503802 & 6.089468 & 5.000000 \\
\hline & 18.684498 & 8.215200 & 5.000000 \\
\hline & 18.684498 & 6.784800 & 5.000000 \\
\hline & 3.025157 & 8.751624 & 5.000000 \\
\hline & 3.025157 & 6.248376 & 5.000000 \\
\hline & 5.158323 & 10.002799 & 5.000000 \\
\hline & 5.158323 & 4.997201 & 5.000000 \\
\hline & 7.630319 & 10.003859 & 5.000000 \\
\hline & 7.630319 & 4.996141 & 5.000000 \\
\hline & 10.097555 & 10.005150 & 5.000000 \\
\hline & 10.097555 & 4.994850 & 5.000000 \\
\hline & 12.562447 & 10.005150 & 5.000000 \\
\hline & 12.562447 & 4.994850 & 5.000000 \\
\hline & 15.029684 & 10.003859 & 5.000000 \\
\hline & 15.029684 & 4.996141 & 5.000000 \\
\hline & 17.501680 & 10.002799 & 5.000000 \\
\hline & 17.501680 & 4.997201 & 5.000000 \\
\hline & 19.634845 & 8.751624 & 5.000000 \\
\hline & 19.634845 & 6.248376 & 5.000000 \\
\hline & ene & & \\
\hline & 5.454403 & 19.406083 & 0.000000 \\
\hline C & 5.967398 & 4.605260 & 0.000000 \\
\hline & 5.539857 & 16.931818 & 0.000000 \\
\hline & 5.623374 & 14.466540 & 0.000000 \\
\hline & 5.881678 & 7.077959 & 0.000000 \\
\hline & 5.793127 & 9.543005 & 0.000000 \\
\hline & 5.706931 & 12.005006 & 0.000000 \\
\hline & 8.786177 & 4.701289 & 0.000000 \\
\hline & 8.700640 & 7.176111 & 0.000000 \\
\hline & 8.612915 & 9.641043 & 0.000000 \\
\hline & 8.530526 & 12.102772 & 0.000000 \\
\hline & 8.445259 & 14.564498 & 0.000000 \\
\hline & 8.358342 & 17.029713 & 0.000000 \\
\hline & 8.273522 & 19.503624 & 0.000000 \\
\hline & 6.702544 & 3.447638 & 0.000000 \\
\hline & 8.131149 & 3.495838 & 0.000000 \\
\hline & 6.606342 & 5.886558 & 0.000000 \\
\hline$\checkmark$ & 8.061556 & 5.936540 & 0.000000 \\
\hline $\mathrm{C}$ & 6.516489 & 8.340623 & 0.000000 \\
\hline C & 7.977030 & 8.390964 & 0.000000 \\
\hline 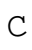 & 6.429174 & 10.798980 & 0.000000 \\
\hline C & 7.892469 & 10.849909 & 0.000000 \\
\hline & 6.344404 & 13.258075 & 0.000000 \\
\hline C & 7.808347 & 13.308837 & 0.000000 \\
\hline C & 6.261858 & 15.716436 & 0.000000 \\
\hline C & 7.722535 & 15.767162 & 0.000000 \\
\hline & 6.179365 & 18.171150 & 0.000000 \\
\hline & 7.634513 & 18.221828 & 0.000000 \\
\hline & 6.109053 & 20.611585 & 0.000000 \\
\hline & 7.537526 & 20.661118 & 0.000000 \\
\hline & 6.197309 & 2.480841 & 0.000000 \\
\hline
\end{tabular}




\begin{tabular}{|c|c|c|c|}
\hline $\mathrm{H}$ & 8.698967 & 2.563864 & 0.000000 \\
\hline $\mathrm{H}$ & 4.875191 & 4.570416 & 0.000000 \\
\hline $\mathrm{H}$ & 9.878361 & 4.740701 & 0.000000 \\
\hline $\mathrm{H}$ & 4.788759 & 7.039438 & 0.000000 \\
\hline $\mathrm{H}$ & 9.793721 & 7.215288 & 0.000000 \\
\hline $\mathrm{H}$ & 4.700490 & 9.505488 & 0.000000 \\
\hline $\mathrm{H}$ & 9.705859 & 9.680041 & 0.000000 \\
\hline $\mathrm{H}$ & 4.614365 & 11.967264 & 0.000000 \\
\hline $\mathrm{H}$ & 9.623092 & 12.140601 & 0.000000 \\
\hline $\mathrm{H}$ & 4.530783 & 14.429464 & 0.000000 \\
\hline $\mathrm{H}$ & 9.538023 & 14.601199 & 0.000000 \\
\hline $\mathrm{H}$ & 4.446881 & 16.893684 & 0.000000 \\
\hline $\mathrm{H}$ & 9.451393 & 17.067208 & 0.000000 \\
\hline $\mathrm{H}$ & 4.362259 & 19.366076 & 0.000000 \\
\hline $\mathrm{H}$ & 5.541259 & 21.543625 & 0.000000 \\
\hline $\mathrm{H}$ & 8.038972 & 21.630441 & 0.000000 \\
\hline $\mathrm{H}$ & 9.365803 & 19.538422 & 0.000000 \\
\hline \multicolumn{4}{|c|}{ [8]-acene } \\
\hline C & 6.490371 & 6.829062 & 5.655684 \\
\hline $\mathrm{C}$ & 6.490371 & 9.304816 & 5.665078 \\
\hline $\mathrm{C}$ & 6.489030 & 11.771807 & 5.674598 \\
\hline $\mathrm{C}$ & 6.489012 & 14.234748 & 5.682959 \\
\hline C & 6.489614 & 16.695823 & 5.693204 \\
\hline $\mathrm{C}$ & 6.490555 & 19.158813 & 5.706263 \\
\hline C & 6.491993 & 21.625554 & 5.719564 \\
\hline $\mathrm{C}$ & 6.491269 & 24.101280 & 5.731605 \\
\hline $\mathrm{C}$ & 9.308026 & 6.829644 & 5.657939 \\
\hline C & 9.307866 & 9.304755 & 5.667490 \\
\hline $\mathrm{C}$ & 9.310383 & 11.771392 & 5.677572 \\
\hline C & 9.311991 & 14.234130 & 5.686494 \\
\hline C & 9.312499 & 16.695665 & 5.696653 \\
\hline C & 9.311760 & 19.158901 & 5.709295 \\
\hline C & 9.309577 & 21.626109 & 5.722107 \\
\hline C & 9.309018 & 24.101543 & 5.734037 \\
\hline $\mathrm{C}$ & 7.187189 & 5.643914 & 5.651851 \\
\hline C & 8.612355 & 5.644314 & 5.653215 \\
\hline C & 7.173182 & 8.083093 & 5.660878 \\
\hline C & 8.625014 & 8.083274 & 5.661949 \\
\hline C & 7.170114 & 10.538559 & 5.670526 \\
\hline C & 8.628844 & 10.538538 & 5.671682 \\
\hline $\mathrm{C}$ & 7.168351 & 13.000117 & 5.679024 \\
\hline C & 8.631960 & 12.999952 & 5.680854 \\
\hline C & 7.168477 & 15.465196 & 5.687773 \\
\hline $\mathrm{C}$ & 8.633632 & 15.465023 & 5.689684 \\
\hline C & 7.169422 & 17.930342 & 5.699744 \\
\hline $\mathrm{C}$ & 8.632982 & 17.930357 & 5.701382 \\
\hline C & 7.171868 & 20.392007 & 5.713535 \\
\hline C & 8.630646 & 20.392171 & 5.714733 \\
\hline C & 7.174565 & 22.847359 & 5.725943 \\
\hline C & 8.626510 & 22.847521 & 5.727005 \\
\hline C & 7.187452 & 25.286569 & 5.737782 \\
\hline C & 8.612702 & 25.286867 & 5.739042 \\
\hline $\mathrm{H}$ & 6.650691 & 4.693750 & 5.647894 \\
\hline $\mathrm{H}$ & 9.150022 & 4.694759 & 5.650317 \\
\hline $\mathrm{H}$ & 5.398088 & 6.833814 & 5.655275 \\
\hline $\mathrm{H}$ & 10.400482 & 6.835061 & 5.65931 \\
\hline $\mathrm{H}$ & 5.396862 & 9.304561 & 5.66499 \\
\hline
\end{tabular}




\begin{tabular}{|c|c|c|c|}
\hline $\mathrm{H}$ & 10.401528 & 9.304142 & 5.669512 \\
\hline $\mathrm{H}$ & 5.395590 & 11.770880 & 5.674726 \\
\hline $\mathrm{H}$ & 10.403917 & 11.769968 & 5.680246 \\
\hline $\mathrm{H}$ & 5.395739 & 14.234785 & 5.683993 \\
\hline $\mathrm{H}$ & 10.405359 & 14.233270 & 5.689992 \\
\hline $\mathrm{H}$ & 5.396318 & 16.696661 & 5.694241 \\
\hline $\mathrm{H}$ & 10.405909 & 16.696256 & 5.700215 \\
\hline $\mathrm{H}$ & 5.397060 & 19.159929 & 5.706323 \\
\hline $\mathrm{H}$ & 10.405274 & 19.159945 & 5.712160 \\
\hline $\mathrm{H}$ & 5.398439 & 21.625587 & 5.719080 \\
\hline $\mathrm{H}$ & 10.403161 & 21.626497 & 5.723838 \\
\hline $\mathrm{H}$ & 5.398897 & 24.095903 & 5.730652 \\
\hline $\mathrm{H}$ & 10.401439 & 24.096370 & 5.735182 \\
\hline $\mathrm{H}$ & 6.650847 & 26.236809 & 5.741274 \\
\hline $\mathrm{H}$ & 9.149834 & 26.236716 & 5.743754 \\
\hline \multicolumn{4}{|c|}{ [9]-acene } \\
\hline C & 16.610001 & 6.088793 & 5.000000 \\
\hline C & 14.150877 & 6.088920 & 5.000000 \\
\hline $\mathrm{C}$ & 19.069122 & 6.088920 & 5.000000 \\
\hline C & 11.689621 & 6.089299 & 5.000000 \\
\hline C & 21.530378 & 6.089299 & 5.000000 \\
\hline $\mathrm{C}$ & 9.223705 & 6.091455 & 5.000000 \\
\hline $\mathrm{C}$ & 23.996297 & 6.091455 & 5.000000 \\
\hline $\mathrm{C}$ & 6.747861 & 6.091554 & 5.000000 \\
\hline C & 26.472138 & 6.091554 & 5.000000 \\
\hline $\mathrm{C}$ & 15.377450 & 6.767573 & 5.000000 \\
\hline $\mathrm{C}$ & 17.842552 & 6.767573 & 5.000000 \\
\hline C & 12.913948 & 6.768757 & 5.000000 \\
\hline $\mathrm{C}$ & 20.306051 & 6.768757 & 5.000000 \\
\hline $\mathrm{C}$ & 10.454781 & 6.771045 & 5.000000 \\
\hline C & 22.765221 & 6.771045 & 5.000000 \\
\hline C & 8.000547 & 6.774578 & 5.000000 \\
\hline $\mathrm{C}$ & 25.219449 & 6.774578 & 5.000000 \\
\hline C & 5.562188 & 6.788197 & 5.000000 \\
\hline $\mathrm{C}$ & 27.657811 & 6.788197 & 5.000000 \\
\hline $\mathrm{C}$ & 5.562188 & 8.211803 & 5.000000 \\
\hline C & 27.657811 & 8.211803 & 5.000000 \\
\hline $\mathrm{C}$ & 8.000547 & 8.225423 & 5.000000 \\
\hline $\mathrm{C}$ & 25.219449 & 8.225423 & 5.000000 \\
\hline C & 10.454781 & 8.228953 & 5.000000 \\
\hline $\mathrm{C}$ & 22.765221 & 8.228953 & 5.000000 \\
\hline $\mathrm{C}$ & 12.913948 & 8.231243 & 5.000000 \\
\hline $\mathrm{C}$ & 20.306051 & 8.231243 & 5.000000 \\
\hline $\mathrm{C}$ & 15.377450 & 8.232427 & 5.000000 \\
\hline C & 17.842552 & 8.232427 & 5.000000 \\
\hline $\mathrm{C}$ & 6.747861 & 8.908448 & 5.000000 \\
\hline $\mathrm{C}$ & 26.472138 & 8.908448 & 5.000000 \\
\hline $\mathrm{C}$ & 9.223705 & 8.908545 & 5.000000 \\
\hline $\mathrm{C}$ & 23.996297 & 8.908545 & 5.000000 \\
\hline $\mathrm{C}$ & 11.689621 & 8.910701 & 5.000000 \\
\hline $\mathrm{C}$ & 21.530378 & 8.910701 & 5.000000 \\
\hline $\mathrm{C}$ & 14.150877 & 8.911080 & 5.000000 \\
\hline $\mathrm{C}$ & 19.069122 & 8.911080 & 5.000000 \\
\hline $\mathrm{C}$ & 16.610001 & 8.911207 & 5.000000 \\
\hline $\mathrm{H}$ & 4.613378 & 8.750366 & 5.000000 \\
\hline $\mathrm{H}$ & 4.613378 & 6.249634 & 5.000000 \\
\hline $\mathrm{H}$ & 6.749299 & 10.000887 & 5.000000 \\
\hline
\end{tabular}




\begin{tabular}{|c|c|c|c|}
\hline $\mathrm{H}$ & 6.749299 & 4.999115 & 5.000000 \\
\hline $\mathrm{H}$ & 9.222114 & 10.002104 & 5.000000 \\
\hline $\mathrm{H}$ & 9.222114 & 4.997896 & 5.000000 \\
\hline $\mathrm{H}$ & 11.688285 & 10.004017 & 5.000000 \\
\hline $\mathrm{H}$ & 11.688285 & 4.995985 & 5.000000 \\
\hline $\mathrm{H}$ & 14.150240 & 10.004581 & 5.000000 \\
\hline $\mathrm{H}$ & 14.150240 & 4.995419 & 5.000000 \\
\hline $\mathrm{H}$ & 16.610001 & 10.004794 & 5.000000 \\
\hline $\mathrm{H}$ & 16.610001 & 4.995206 & 5.000000 \\
\hline $\mathrm{H}$ & 19.069759 & 10.004581 & 5.000000 \\
\hline $\mathrm{H}$ & 19.069759 & 4.995419 & 5.000000 \\
\hline $\mathrm{H}$ & 21.531713 & 10.004017 & 5.000000 \\
\hline $\mathrm{H}$ & 21.531713 & 4.995985 & 5.000000 \\
\hline $\mathrm{H}$ & 23.997884 & 10.002104 & 5.000000 \\
\hline $\mathrm{H}$ & 23.997884 & 4.997896 & 5.000000 \\
\hline $\mathrm{H}$ & 26.470700 & 10.000887 & 5.000000 \\
\hline $\mathrm{H}$ & 26.470700 & 4.999115 & 5.000000 \\
\hline $\mathrm{H}$ & 28.606621 & 8.750366 & 5.000000 \\
\hline $\mathrm{H}$ & 28.606621 & 6.249634 & 5.000000 \\
\hline \multicolumn{4}{|c|}{ [10] - acene } \\
\hline $\mathrm{C}$ & 18.961729 & 5.503042 & 4.005200 \\
\hline C & 16.503418 & 5.502992 & 4.005200 \\
\hline $\mathrm{C}$ & 14.043658 & 5.503106 & 4.005200 \\
\hline C & 21.421622 & 5.502856 & 4.005200 \\
\hline C & 23.884579 & 5.503772 & 4.005200 \\
\hline C & 11.581062 & 5.503988 & 4.005200 \\
\hline C & 26.351539 & 5.505922 & 4.005200 \\
\hline C & 9.114821 & 5.505650 & 4.005200 \\
\hline $\mathrm{C}$ & 6.639440 & 5.504119 & 4.005200 \\
\hline $\mathrm{C}$ & 28.827044 & 5.505034 & 4.005200 \\
\hline C & 17.732564 & 6.181390 & 4.005200 \\
\hline C & 20.197433 & 6.181432 & 4.005200 \\
\hline C & 15.267825 & 6.181561 & 4.005200 \\
\hline C & 22.661197 & 6.182435 & 4.005200 \\
\hline $\mathrm{C}$ & 12.804309 & 6.182736 & 4.005200 \\
\hline $\mathrm{C}$ & 25.120894 & 6.185199 & 4.005200 \\
\hline C & 10.345008 & 6.185490 & 4.005200 \\
\hline $\mathrm{C}$ & 27.574782 & 6.188636 & 4.005200 \\
\hline C & 7.891429 & 6.188013 & 4.005200 \\
\hline $\mathrm{C}$ & 30.012844 & 6.200875 & 4.005200 \\
\hline $\mathrm{C}$ & 5.453400 & 6.199604 & 4.005200 \\
\hline $\mathrm{C}$ & 5.452794 & 7.623542 & 4.005200 \\
\hline C & 30.012980 & 7.624851 & 4.005200 \\
\hline C & 27.574885 & 7.638887 & 4.005200 \\
\hline $\mathrm{C}$ & 7.890827 & 7.638344 & 4.005200 \\
\hline $\mathrm{C}$ & 25.120977 & 7.641756 & 4.005200 \\
\hline $\mathrm{C}$ & 10.344838 & 7.642003 & 4.005200 \\
\hline C & 22.661190 & 7.643781 & 4.005200 \\
\hline C & 20.197569 & 7.645271 & 4.005200 \\
\hline C & 12.804323 & 7.644006 & 4.005200 \\
\hline $\mathrm{C}$ & 15.267860 & 7.645405 & 4.005200 \\
\hline $\mathrm{C}$ & 17.732625 & 7.645884 & 4.005200 \\
\hline $\mathrm{C}$ & 6.637883 & 8.321024 & 4.005200 \\
\hline $\mathrm{C}$ & 28.827669 & 8.321803 & 4.005200 \\
\hline $\mathrm{C}$ & 26.351508 & 8.321761 & 4.005200 \\
\hline C & 9.114131 & 8.321375 & 4.005200 \\
\hline C & 11.581243 & 8.323449 & 4.005200 \\
\hline
\end{tabular}




\begin{tabular}{|c|c|c|c|}
\hline C & 23.884449 & 8.322879 & 4.005200 \\
\hline$z$ & 21.421812 & 8.323849 & 4.005200 \\
\hline C & 14.043666 & 8.323820 & 4.005200 \\
\hline C & 18.962000 & 8.324106 & 4.005200 \\
\hline C & 16.503427 & 8.324411 & 4.005200 \\
\hline $\mathrm{H}$ & 4.502735 & 8.159581 & 4.005200 \\
\hline $\mathrm{H}$ & 4.504093 & 5.662182 & 4.005200 \\
\hline $\mathrm{H}$ & 6.640589 & 9.413287 & 4.005200 \\
\hline $\mathrm{H}$ & 6.643877 & 4.411796 & 4.005200 \\
\hline $\mathrm{H}$ & 9.112948 & 9.414502 & 4.005200 \\
\hline $\mathrm{H}$ & 9.114178 & 4.412444 & 4.005200 \\
\hline $\mathrm{H}$ & 11.581278 & 9.416445 & 4.005200 \\
\hline $\mathrm{H}$ & 11.580248 & 4.410890 & 4.005200 \\
\hline $\mathrm{H}$ & 14.043984 & 9.416943 & 4.005200 \\
\hline $\mathrm{H}$ & 14.044299 & 4.409876 & 4.005200 \\
\hline $\mathrm{H}$ & 16.503308 & 9.417509 & 4.005200 \\
\hline $\mathrm{H}$ & 16.503673 & 4.409757 & 4.005200 \\
\hline $\mathrm{H}$ & 18.961880 & 9.417224 & 4.005200 \\
\hline $\mathrm{H}$ & 18.961390 & 4.409816 & 4.005200 \\
\hline $\mathrm{H}$ & 21.421143 & 9.416941 & 4.005200 \\
\hline $\mathrm{H}$ & 21.420583 & 4.409598 & 4.005200 \\
\hline $\mathrm{H}$ & 23.884007 & 9.415888 & 4.005200 \\
\hline $\mathrm{H}$ & 23.885088 & 4.410644 & 4.005200 \\
\hline $\mathrm{H}$ & 26.352604 & 9.414899 & 4.005200 \\
\hline $\mathrm{H}$ & 26.353103 & 4.412717 & 4.005200 \\
\hline $\mathrm{H}$ & 28.824082 & 9.414075 & 4.005200 \\
\hline $\mathrm{H}$ & 30.962953 & 8.161176 & 4.005200 \\
\hline $\mathrm{H}$ & 30.962537 & 5.664182 & 4.005200 \\
\hline $\mathrm{H}$ & 28.822431 & 4.412739 & 4.005200 \\
\hline \multicolumn{4}{|c|}{ vene } \\
\hline C & 7.806266 & 6.091637 & 5.000000 \\
\hline C & 10.282698 & 6.091871 & 5.000000 \\
\hline C & 12.749464 & 6.090000 & 5.000000 \\
\hline C & 15.211787 & 6.089442 & 5.000000 \\
\hline C & 17.671413 & 6.089744 & 5.000000 \\
\hline C & 20.129999 & 6.089614 & 5.000000 \\
\hline C & 22.588590 & 6.089744 & 5.000000 \\
\hline C & 25.048216 & 6.089442 & 5.000000 \\
\hline C & 27.510534 & 6.090000 & 5.000000 \\
\hline C & 29.977300 & 6.091871 & 5.000000 \\
\hline C & 32.453729 & 6.091637 & 5.000000 \\
\hline C & 6.620794 & 6.787885 & 5.000000 \\
\hline C & 9.059588 & 6.774518 & 5.000000 \\
\hline C & 11.513607 & 6.771619 & 5.000000 \\
\hline C & 13.972182 & 6.769110 & 5.000000 \\
\hline C & 16.434039 & 6.768387 & 5.000000 \\
\hline C & 18.897843 & 6.767779 & 5.000000 \\
\hline C & 21.362159 & 6.767779 & 5.000000 \\
\hline C & 23.825963 & 6.768387 & 5.000000 \\
\hline $\mathrm{C}$ & 26.287817 & 6.769110 & 5.000000 \\
\hline C & 28.746392 & 6.771619 & 5.000000 \\
\hline C & 31.200407 & 6.774518 & 5.000000 \\
\hline C & 33.639201 & 6.787885 & 5.000000 \\
\hline C & 6.620794 & 8.212115 & 5.000000 \\
\hline C & 9.059588 & 8.225482 & 5.000000 \\
\hline C & 11.513607 & 8.228381 & 5.000000 \\
\hline $\mathrm{C}$ & 13.972182 & 8.230890 & 5.000000 \\
\hline
\end{tabular}




\begin{tabular}{|c|c|c|c|}
\hline C & 16.434039 & 8.231613 & 5.000000 \\
\hline & 18.897843 & 8.232221 & 5.000000 \\
\hline C & 21.362159 & 8.232221 & 5.000000 \\
\hline C & 23.825963 & 8.231613 & 5.000000 \\
\hline C & 26.287817 & 8.230890 & 5.000000 \\
\hline C & 28.746392 & 8.228381 & 5.000000 \\
\hline C & 31.200407 & 8.225482 & 5.000000 \\
\hline & 33.639201 & 8.212115 & 5.000000 \\
\hline & 7.806266 & 8.908363 & 5.000000 \\
\hline & 10.282698 & 8.908129 & 5.000000 \\
\hline & 12.749464 & 8.910000 & 5.000000 \\
\hline & 15.211787 & 8.910558 & 5.000000 \\
\hline & 17.671413 & 8.910256 & 5.000000 \\
\hline & 20.129999 & 8.910386 & 5.000000 \\
\hline & 22.588590 & 8.910256 & 5.000000 \\
\hline & 25.048216 & 8.910558 & 5.000000 \\
\hline & 27.510534 & 8.910000 & 5.000000 \\
\hline & 29.977300 & 8.908129 & 5.000000 \\
\hline & 32.453729 & 8.908363 & 5.000000 \\
\hline & 5.671951 & 8.750513 & 5.000000 \\
\hline & 5.671951 & 6.249487 & 5.000000 \\
\hline $\mathrm{H}$ & 7.808835 & 10.000838 & 5.000000 \\
\hline & 7.808835 & 4.999162 & 5.000000 \\
\hline $\mathrm{H}$ & 10.282466 & 10.001689 & 5.000000 \\
\hline & 10.282466 & 4.998311 & 5.000000 \\
\hline & 12.749200 & 10.003353 & 5.000000 \\
\hline & 12.749200 & 4.996647 & 5.000000 \\
\hline $\mathrm{H}$ & 15.211951 & 10.003923 & 5.000000 \\
\hline & 15.211951 & 4.996077 & 5.000000 \\
\hline & 17.671382 & 10.003764 & 5.000000 \\
\hline & 17.671382 & 4.996236 & 5.000000 \\
\hline & 20.129999 & 10.003926 & 5.000000 \\
\hline & 20.129999 & 4.996074 & 5.000000 \\
\hline $\mathrm{H}$ & 22.588621 & 10.003764 & 5.000000 \\
\hline & 22.588621 & 4.996236 & 5.000000 \\
\hline & 25.048051 & 10.003923 & 5.000000 \\
\hline & 25.048051 & 4.996077 & 5.000000 \\
\hline & 27.510798 & 10.003353 & 5.000000 \\
\hline & 27.510798 & 4.996647 & 5.000000 \\
\hline $\mathrm{H}$ & 29.977532 & 10.001689 & 5.000000 \\
\hline $\mathrm{H}$ & 29.977532 & 4.998311 & 5.000000 \\
\hline $\mathrm{H}$ & 32.451159 & 10.000838 & 5.000000 \\
\hline & 32.451159 & 4.999162 & 5.000000 \\
\hline & 34.588044 & 8.750513 & 5.000000 \\
\hline $\mathrm{H}$ & 34.588044 & 6.249487 & 5.000000 \\
\hline \multicolumn{4}{|c|}{ [12] acene } \\
\hline C & 14.652884 & 5.624105 & 4.485700 \\
\hline & 17.112967 & 5.624898 & 4.485700 \\
\hline & 24.487140 & 5.624920 & 4.485700 \\
\hline 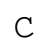 & 26.946440 & 5.624705 & 4.485700 \\
\hline C & 12.189810 & 5.624975 & 4.485700 \\
\hline C & 22.029031 & 5.625675 & 4.485700 \\
\hline $\mathrm{C}$ & 19.571080 & 5.625842 & 4.485700 \\
\hline & 29.408342 & 5.625602 & 4.485700 \\
\hline & 9.722092 & 5.627744 & 4.485700 \\
\hline & 31.875222 & 5.627732 & 4.485700 \\
\hline & 34.352017 & 5.628081 & 4.485700 \\
\hline
\end{tabular}




\begin{tabular}{|c|c|c|c|}
\hline C & 7.245077 & 5.628380 & 4.485700 \\
\hline C & 20.800281 & 6.303829 & 4.485700 \\
\hline C & 23.263288 & 6.303602 & 4.485700 \\
\hline C & 15.874510 & 6.303239 & 4.485700 \\
\hline C & 18.337100 & 6.303826 & 4.485700 \\
\hline C & 25.725297 & 6.303781 & 4.485700 \\
\hline C & 13.413188 & 6.303968 & 4.485700 \\
\hline$c$ & 28.185552 & 6.305038 & 4.485700 \\
\hline C & 10.954419 & 6.306632 & 4.485700 \\
\hline & 30.643255 & 6.307214 & 4.485700 \\
\hline C & 33.097479 & 6.310440 & 4.485700 \\
\hline C & 8.499464 & 6.310601 & 4.485700 \\
\hline C & 35.536742 & 6.324816 & 4.485700 \\
\hline C & 6.060115 & 6.325078 & 4.485700 \\
\hline & 35.536306 & 7.749850 & 4.485700 \\
\hline z & 6.060532 & 7.749942 & 4.485700 \\
\hline & 33.097128 & 7.762354 & 4.485700 \\
\hline & 8.499863 & 7.762597 & 4.485700 \\
\hline C & 10.954737 & 7.764133 & 4.485700 \\
\hline & 30.643037 & 7.764575 & 4.485700 \\
\hline & 13.413291 & 7.765335 & 4.485700 \\
\hline C & 28.185439 & 7.766279 & 4.485700 \\
\hline C & 15.874448 & 7.766674 & 4.485700 \\
\hline & 25.725278 & 7.767126 & 4.485700 \\
\hline & 23.263272 & 7.767868 & 4.485700 \\
\hline & 18.336961 & 7.768112 & 4.485700 \\
\hline & 20.800224 & 7.768379 & 4.485700 \\
\hline C & 9.723102 & 8.444372 & 4.485700 \\
\hline & 31.874659 & 8.444562 & 4.485700 \\
\hline & 12.190439 & 8.445040 & 4.485700 \\
\hline & 14.652762 & 8.445444 & 4.485700 \\
\hline 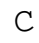 & 34.351120 & 8.445684 & 4.485700 \\
\hline C & 29.408072 & 8.445955 & 4.485700 \\
\hline C & 7.245956 & 8.445903 & 4.485700 \\
\hline C & 26.946284 & 8.446399 & 4.485700 \\
\hline C & 17.112369 & 8.446107 & 4.485700 \\
\hline$\gamma$ & 24.487287 & 8.446201 & 4.485700 \\
\hline$c$ & 22.029161 & 8.446249 & 4.485700 \\
\hline C & 19.570934 & 8.446405 & 4.485700 \\
\hline $\mathrm{H}$ & 5.111396 & 8.287887 & 4.485700 \\
\hline $\mathrm{H}$ & 5.110702 & 5.787612 & 4.485700 \\
\hline $\mathrm{H}$ & 7.247120 & 9.538450 & 4.485700 \\
\hline $\mathrm{H}$ & 7.245746 & 4.535833 & 4.485700 \\
\hline $\mathrm{H}$ & 9.724580 & 9.537939 & 4.485700 \\
\hline $\mathrm{H}$ & 9.722259 & 4.534223 & 4.485700 \\
\hline $\mathrm{H}$ & 12.191779 & 9.538385 & 4.485700 \\
\hline $\mathrm{H}$ & 12.190328 & 4.531597 & 4.485700 \\
\hline $\mathrm{H}$ & 14.652861 & 9.538799 & 4.485700 \\
\hline $\mathrm{H}$ & 14.653364 & 4.530738 & 4.485700 \\
\hline $\mathrm{H}$ & 17.111446 & 9.539585 & 4.485700 \\
\hline $\mathrm{H}$ & 17.113302 & 4.531442 & 4.485700 \\
\hline $\mathrm{H}$ & 19.570635 & 9.540012 & 4.485700 \\
\hline $\mathrm{H}$ & 19.570719 & 4.532302 & 4.485700 \\
\hline $\mathrm{H}$ & 22.029214 & 9.539812 & 4.485700 \\
\hline 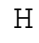 & 22.028840 & 4.532131 & 4.485700 \\
\hline $\mathrm{H}$ & 24.487660 & 9.539645 & 4.485700 \\
\hline $\mathrm{H}$ & 24.487176 & 4.531499 & 4.485700 \\
\hline
\end{tabular}




\begin{tabular}{|c|c|c|c|}
\hline $\mathrm{H}$ & 26.945671 & 9.539748 & 4.485700 \\
\hline $\mathrm{H}$ & 26.946369 & 4.531365 & 4.485700 \\
\hline $\mathrm{H}$ & 29.407042 & 9.539315 & 4.485700 \\
\hline $\mathrm{H}$ & 29.407144 & 4.532259 & 4.485700 \\
\hline $\mathrm{H}$ & 31.873805 & 9.538059 & 4.485700 \\
\hline $\mathrm{H}$ & 31.874801 & 4.534229 & 4.485700 \\
\hline $\mathrm{H}$ & 34.350169 & 9.538212 & 4.485700 \\
\hline $\mathrm{H}$ & 36.485578 & 8.287600 & 4.485700 \\
\hline $\mathrm{H}$ & 36.486353 & 5.787608 & 4.485700 \\
\hline $\mathrm{H}$ & 34.351842 & 4.535505 & 4.485700 \\
\hline \multicolumn{4}{|c|}{ [13] acene } \\
\hline C & 16.473985 & 6.302974 & 0.050000 \\
\hline C & 33.708498 & 6.302892 & 0.050000 \\
\hline C & 18.938928 & 6.303088 & 0.050000 \\
\hline C & 36.173443 & 6.303398 & 0.050000 \\
\hline C & 31.245757 & 6.303616 & 0.050000 \\
\hline C & 21.401350 & 6.304031 & 0.050000 \\
\hline C & 28.784618 & 6.304588 & 0.050000 \\
\hline C & 14.005523 & 6.304883 & 0.050000 \\
\hline C & 23.862821 & 6.304996 & 0.050000 \\
\hline C & 26.323671 & 6.305173 & 0.050000 \\
\hline C & 38.641521 & 6.305680 & 0.050000 \\
\hline C & 11.528812 & 6.305968 & 0.050000 \\
\hline C & 41.118482 & 6.306912 & 0.050000 \\
\hline $\mathrm{C}$ & 30.021240 & 6.981863 & 0.050000 \\
\hline C & 32.485753 & 6.981500 & 0.050000 \\
\hline C & 27.556266 & 6.982275 & 0.050000 \\
\hline C & 22.626101 & 6.982208 & 0.050000 \\
\hline C & 20.161490 & 6.981933 & 0.050000 \\
\hline C & 25.091169 & 6.982572 & 0.050000 \\
\hline C & 17.698457 & 6.982134 & 0.050000 \\
\hline C & 34.948732 & 6.982238 & 0.050000 \\
\hline C & 15.238689 & 6.984077 & 0.050000 \\
\hline C & 37.408503 & 6.984837 & 0.050000 \\
\hline C & 12.783726 & 6.987988 & 0.050000 \\
\hline C & 39.863473 & 6.988825 & 0.050000 \\
\hline C & 10.344483 & 7.003148 & 0.050000 \\
\hline C & 42.302890 & 7.004260 & 0.050000 \\
\hline C & 10.345040 & 8.428240 & 0.050000 \\
\hline C & 42.302118 & 8.429411 & 0.050000 \\
\hline C & 12.784253 & 8.439661 & 0.050000 \\
\hline C & 39.862762 & 8.440581 & 0.050000 \\
\hline C & 15.239028 & 8.441620 & 0.050000 \\
\hline C & 37.407989 & 8.442239 & 0.050000 \\
\hline C & 17.698428 & 8.443660 & 0.050000 \\
\hline C & 34.948513 & 8.443721 & 0.050000 \\
\hline C & 32.485838 & 8.444883 & 0.050000 \\
\hline C & 20.161299 & 8.445232 & 0.050000 \\
\hline C & 30.021435 & 8.445661 & 0.050000 \\
\hline C & 22.626042 & 8.446096 & 0.050000 \\
\hline C & 27.556306 & 8.446182 & 0.050000 \\
\hline C & 25.091179 & 8.446442 & 0.050000 \\
\hline C & 14.006528 & 9.121424 & 0.050000 \\
\hline C & 38.640177 & 9.122212 & 0.050000 \\
\hline C & 16.474254 & 9.122549 & 0.050000 \\
\hline C & 36.172676 & 9.122819 & 0.050000 \\
\hline C & 33.708330 & 9.123101 & 0.05000 \\
\hline
\end{tabular}




\begin{tabular}{|c|c|c|c|}
\hline C & 18.938701 & 9.123179 & 0.050000 \\
\hline 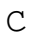 & 31.245947 & 9.123129 & 0.050000 \\
\hline & 28.784817 & 9.123119 & 0.050000 \\
\hline$C$ & 21.401161 & 9.123433 & 0.050000 \\
\hline C & 11.530667 & 9.123437 & 0.050000 \\
\hline C & 26.323828 & 9.123375 & 0.050000 \\
\hline C & 23.862732 & 9.123500 & 0.050000 \\
\hline C & 41.116491 & 9.124394 & 0.050000 \\
\hline $\mathrm{H}$ & 9.395499 & 8.965564 & 0.050000 \\
\hline H & 9.393944 & 6.467337 & 0.050000 \\
\hline $\mathrm{H}$ & 11.534098 & 10.215966 & 0.050000 \\
\hline $\mathrm{H}$ & 11.530466 & 5.213457 & 0.050000 \\
\hline $\mathrm{H}$ & 14.005863 & 10.214993 & 0.050000 \\
\hline $\mathrm{H}$ & 14.003750 & 5.211391 & 0.050000 \\
\hline $\mathrm{H}$ & 16.473631 & 10.215938 & 0.050000 \\
\hline $\mathrm{H}$ & 16.473230 & 5.209608 & 0.050000 \\
\hline $\mathrm{H}$ & 18.938716 & 10.216576 & 0.050000 \\
\hline $\mathrm{H}$ & 18.939519 & 5.209680 & 0.050000 \\
\hline $\mathrm{H}$ & 21.401699 & 10.216928 & 0.050000 \\
\hline $\mathrm{H}$ & 21.402254 & 5.210547 & 0.050000 \\
\hline $\mathrm{H}$ & 23.863195 & 10.217063 & 0.050000 \\
\hline $\mathrm{H}$ & 23.863576 & 5.211441 & 0.050000 \\
\hline $\mathrm{H}$ & 26.323959 & 10.216953 & 0.050000 \\
\hline $\mathrm{H}$ & 26.323509 & 5.211600 & 0.050000 \\
\hline $\mathrm{H}$ & 28.784569 & 10.216678 & 0.050000 \\
\hline $\mathrm{H}$ & 28.783966 & 5.211044 & 0.050000 \\
\hline $\mathrm{H}$ & 31.245284 & 10.216615 & 0.050000 \\
\hline $\mathrm{H}$ & 31.244831 & 5.210132 & 0.050000 \\
\hline $\mathrm{H}$ & 33.707913 & 10.216518 & 0.050000 \\
\hline $\mathrm{H}$ & 33.708208 & 5.209508 & 0.050000 \\
\hline $\mathrm{H}$ & 36.172906 & 10.216238 & 0.050000 \\
\hline $\mathrm{H}$ & 36.174484 & 5.210049 & 0.050000 \\
\hline $\mathrm{H}$ & 43.251612 & 8.966809 & 0.050000 \\
\hline $\mathrm{H}$ & 43.253189 & 6.468153 & 0.050000 \\
\hline $\mathrm{H}$ & 38.640764 & 10.215777 & 0.050000 \\
\hline $\mathrm{H}$ & 38.643055 & 5.212193 & 0.050000 \\
\hline $\mathrm{H}$ & 41.112818 & 10.216933 & 0.050000 \\
\hline $\mathrm{H}$ & 41.116629 & 5.214377 & 0.050000 \\
\hline \multicolumn{4}{|c|}{ 14] acene } \\
\hline C & 16.497388 & 6.302485 & 0.050000 \\
\hline C & 18.959380 & 6.302961 & 0.050000 \\
\hline C & 21.419933 & 6.304049 & 0.050000 \\
\hline C & 14.033306 & 6.303154 & 0.050000 \\
\hline C & 33.724203 & 6.303044 & 0.050000 \\
\hline C & 36.188606 & 6.303291 & 0.050000 \\
\hline C & 23.880307 & 6.304844 & 0.050000 \\
\hline C & 31.262022 & 6.303679 & 0.050000 \\
\hline C & 28.801026 & 6.304526 & 0.050000 \\
\hline C & 26.340579 & 6.305147 & 0.050000 \\
\hline $\mathrm{C}$ & 11.565675 & 6.305417 & 0.050000 \\
\hline C & 38.656340 & 6.305671 & 0.050000 \\
\hline C & 9.089131 & 6.306718 & 0.050000 \\
\hline C & 41.133018 & 6.306907 & 0.050000 \\
\hline C & 17.720359 & 6.981114 & 0.050000 \\
\hline C & 20.183819 & 6.981407 & 0.050000 \\
\hline C & 22.647442 & 6.982012 & 0.050000 \\
\hline C & 30.037909 & 6.981923 & 0.050000 \\
\hline
\end{tabular}




\begin{tabular}{|c|c|c|c|}
\hline C & 32.501539 & 6.981780 & 0.050000 \\
\hline & 15.258240 & 6.982065 & 0.050000 \\
\hline & 25.110691 & 6.982508 & 0.050000 \\
\hline 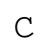 & 27.574183 & 6.982367 & 0.050000 \\
\hline C & 34.963936 & 6.982288 & 0.050000 \\
\hline C & 12.798985 & 6.984458 & 0.050000 \\
\hline & 37.423230 & 6.984613 & 0.050000 \\
\hline & 10.344257 & 6.988475 & 0.050000 \\
\hline & 39.877905 & 6.988654 & 0.050000 \\
\hline & 7.904847 & 7.003906 & 0.050000 \\
\hline & 42.316929 & 7.004060 & 0.050000 \\
\hline & 7.905945 & 8.429273 & 0.050000 \\
\hline & 42.316405 & 8.429392 & 0.050000 \\
\hline & 10.345123 & 8.440518 & 0.050000 \\
\hline & 39.877356 & 8.440712 & 0.050000 \\
\hline & 12.799774 & 8.442053 & 0.050000 \\
\hline & 37.422892 & 8.442215 & 0.050000 \\
\hline & 15.258872 & 8.443655 & 0.050000 \\
\hline & 34.963920 & 8.443895 & 0.050000 \\
\hline & 17.720890 & 8.444526 & 0.050000 \\
\hline & 32.501961 & 8.445178 & 0.050000 \\
\hline & 20.184380 & 8.445039 & 0.050000 \\
\hline & 30.038745 & 8.445616 & 0.050000 \\
\hline & 22.648110 & 8.445558 & 0.050000 \\
\hline & 27.574989 & 8.445891 & 0.050000 \\
\hline & 25.111479 & 8.445932 & 0.050000 \\
\hline & 11.567289 & 9.121938 & 0.050000 \\
\hline & 14.034480 & 9.122667 & 0.050000 \\
\hline & 38.655280 & 9.122221 & 0.050000 \\
\hline & 16.498376 & 9.122938 & 0.050000 \\
\hline & 18.960262 & 9.122751 & 0.050000 \\
\hline & 21.420927 & 9.122679 & 0.050000 \\
\hline & 36.188522 & 9.122919 & 0.050000 \\
\hline & 23.881602 & 9.123002 & 0.050000 \\
\hline & 33.724990 & 9.123398 & 0.050000 \\
\hline & 9.091186 & 9.124152 & 0.050000 \\
\hline & 26.342243 & 9.123082 & 0.050000 \\
\hline & 41.131133 & 9.124471 & 0.050000 \\
\hline & 28.802636 & 9.123130 & 0.050000 \\
\hline C & 31.263337 & 9.123480 & 0.050000 \\
\hline & 6.956478 & 8.966819 & 0.050000 \\
\hline & 6.954600 & 6.467828 & 0.050000 \\
\hline & 9.094654 & 10.216653 & 0.050000 \\
\hline & 9.090620 & 5.214310 & 0.050000 \\
\hline & 11.567330 & 10.215435 & 0.050000 \\
\hline & 11.564436 & 5.211990 & 0.050000 \\
\hline & 14.034566 & 10.216019 & 0.050000 \\
\hline & 14.032524 & 5.209829 & 0.050000 \\
\hline & 16.499129 & 10.216298 & 0.050000 \\
\hline & 16.497243 & 5.209129 & 0.050000 \\
\hline $\mathrm{H}$ & 18.961708 & 10.216174 & 0.050000 \\
\hline $\mathrm{H}$ & 18.959251 & 5.209545 & 0.050000 \\
\hline & 21.422532 & 10.216167 & 0.050000 \\
\hline & 21.419147 & 5.210583 & 0.050000 \\
\hline & 23.883272 & 10.216503 & 0.050000 \\
\hline & 23.878898 & 5.211405 & 0.050000 \\
\hline & 26.343943 & 10.216637 & 0.050000 \\
\hline
\end{tabular}




\begin{tabular}{|c|c|c|c|}
\hline $\mathrm{H}$ & 26.338609 & 5.211747 & 0.050000 \\
\hline $\mathrm{H}$ & 28.803792 & 10.216705 & 0.050000 \\
\hline $\mathrm{H}$ & 28.798904 & 5.211162 & 0.050000 \\
\hline $\mathrm{H}$ & 31.263838 & 10.216994 & 0.050000 \\
\hline $\mathrm{H}$ & 31.260039 & 5.210362 & 0.050000 \\
\hline $\mathrm{H}$ & 33.725441 & 10.216817 & 0.050000 \\
\hline $\mathrm{H}$ & 33.722961 & 5.209747 & 0.050000 \\
\hline $\mathrm{H}$ & 36.188998 & 10.216321 & 0.050000 \\
\hline $\mathrm{H}$ & 36.188783 & 5.210015 & 0.050000 \\
\hline $\mathrm{H}$ & 43.265828 & 8.966778 & 0.050000 \\
\hline $\mathrm{H}$ & 43.267195 & 6.467833 & 0.050000 \\
\hline $\mathrm{H}$ & 38.655420 & 10.215719 & 0.050000 \\
\hline $\mathrm{H}$ & 38.657265 & 5.212236 & 0.050000 \\
\hline $\mathrm{H}$ & 41.127729 & 10.216898 & 0.050000 \\
\hline $\mathrm{H}$ & 41.131314 & 5.214428 & 0.050000 \\
\hline \multicolumn{4}{|c|}{ [15] acene } \\
\hline $\mathrm{C}$ & 36.157147 & 6.301936 & 0.050000 \\
\hline C & 16.490333 & 6.302277 & 0.050000 \\
\hline $\mathrm{C}$ & 14.028005 & 6.302788 & 0.050000 \\
\hline C & 33.697291 & 6.302047 & 0.050000 \\
\hline $\mathrm{C}$ & 38.619219 & 6.302878 & 0.050000 \\
\hline $\mathrm{C}$ & 31.238764 & 6.302920 & 0.050000 \\
\hline $\mathrm{C}$ & 18.950186 & 6.302989 & 0.050000 \\
\hline $\mathrm{C}$ & 11.561819 & 6.305139 & 0.050000 \\
\hline $\mathrm{C}$ & 41.085293 & 6.305425 & 0.050000 \\
\hline $\mathrm{C}$ & 21.408292 & 6.303966 & 0.050000 \\
\hline C & 28.781280 & 6.303874 & 0.050000 \\
\hline C & 26.323710 & 6.304559 & 0.050000 \\
\hline $\mathrm{C}$ & 23.866183 & 6.304701 & 0.050000 \\
\hline $\mathrm{C}$ & 43.560595 & 6.306603 & 0.050000 \\
\hline $\mathrm{C}$ & 9.086180 & 6.306873 & 0.050000 \\
\hline $\mathrm{C}$ & 32.473746 & 6.981001 & 0.050000 \\
\hline $\mathrm{C}$ & 34.934753 & 6.980908 & 0.050000 \\
\hline C & 30.013229 & 6.981896 & 0.050000 \\
\hline $\mathrm{C}$ & 17.712454 & 6.981660 & 0.050000 \\
\hline $\mathrm{C}$ & 27.553477 & 6.982600 & 0.050000 \\
\hline C & 20.173515 & 6.982180 & 0.050000 \\
\hline $\mathrm{C}$ & 22.634157 & 6.982656 & 0.050000 \\
\hline $\mathrm{C}$ & 37.394922 & 6.982083 & 0.050000 \\
\hline $\mathrm{C}$ & 15.252298 & 6.982126 & 0.050000 \\
\hline $\mathrm{C}$ & 25.094059 & 6.983023 & 0.050000 \\
\hline $\mathrm{C}$ & 12.794683 & 6.984383 & 0.050000 \\
\hline C & 39.852512 & 6.984685 & 0.050000 \\
\hline C & 42.305861 & 6.988704 & 0.050000 \\
\hline $\mathrm{C}$ & 10.341099 & 6.988560 & 0.050000 \\
\hline $\mathrm{C}$ & 44.744359 & 7.003874 & 0.050000 \\
\hline $\mathrm{C}$ & 7.902221 & 7.003997 & 0.050000 \\
\hline C & 7.903036 & 8.429580 & 0.050000 \\
\hline C & 44.743569 & 8.429454 & 0.050000 \\
\hline C & 10.341818 & 8.440498 & 0.050000 \\
\hline C & 42.305277 & 8.440716 & 0.050000 \\
\hline C & 12.795099 & 8.442037 & 0.050000 \\
\hline $\mathrm{C}$ & 39.852239 & 8.442369 & 0.050000 \\
\hline C & 37.394789 & 8.443741 & 0.050000 \\
\hline C & 15.252369 & 8.443805 & 0.050000 \\
\hline C & 34.934705 & 8.444349 & 0.050000 \\
\hline $\mathrm{C}$ & 32.473945 & 8.444700 & 0.050000 \\
\hline
\end{tabular}




\begin{tabular}{|c|c|c|c|}
\hline C & 17.712210 & 8.445084 & 0.050000 \\
\hline $\mathrm{C}$ & 30.013375 & 8.445142 & 0.050000 \\
\hline 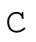 & 20.173396 & 8.445794 & 0.050000 \\
\hline$C$ & 27.553534 & 8.445516 & 0.050000 \\
\hline C & 22.634180 & 8.445938 & 0.050000 \\
\hline C & 25.094074 & 8.445993 & 0.050000 \\
\hline C & 31.239226 & 9.123170 & 0.050000 \\
\hline C & 28.781641 & 9.123383 & 0.050000 \\
\hline C & 11.563067 & 9.122188 & 0.050000 \\
\hline C & 33.697515 & 9.123259 & 0.050000 \\
\hline C & 41.084229 & 9.122608 & 0.050000 \\
\hline C & 26.323917 & 9.123837 & 0.050000 \\
\hline C & 23.866127 & 9.124034 & 0.050000 \\
\hline C & 36.157021 & 9.123347 & 0.050000 \\
\hline C & 14.028286 & 9.123175 & 0.050000 \\
\hline C & 21.408037 & 9.124071 & 0.050000 \\
\hline C & 38.618670 & 9.123245 & 0.050000 \\
\hline C & 18.949720 & 9.124136 & 0.050000 \\
\hline C & 16.489992 & 9.123863 & 0.050000 \\
\hline C & 9.088349 & 9.124366 & 0.050000 \\
\hline C & 43.558877 & 9.124468 & 0.050000 \\
\hline $\mathrm{H}$ & 6.953895 & 8.967215 & 0.050000 \\
\hline $\mathrm{H}$ & 6.952153 & 6.467797 & 0.050000 \\
\hline $\mathrm{H}$ & 9.093294 & 10.216930 & 0.050000 \\
\hline $\mathrm{H}$ & 9.089129 & 5.214244 & 0.050000 \\
\hline $\mathrm{H}$ & 11.563768 & 10.215775 & 0.050000 \\
\hline $\mathrm{H}$ & 11.561334 & 5.211626 & 0.050000 \\
\hline $\mathrm{H}$ & 14.028263 & 10.216568 & 0.050000 \\
\hline $\mathrm{H}$ & 14.027710 & 5.209442 & 0.050000 \\
\hline $\mathrm{H}$ & 16.490067 & 10.217228 & 0.050000 \\
\hline $\mathrm{H}$ & 16.490970 & 5.208981 & 0.050000 \\
\hline $\mathrm{H}$ & 18.950424 & 10.217535 & 0.050000 \\
\hline $\mathrm{H}$ & 18.951458 & 5.209655 & 0.050000 \\
\hline $\mathrm{H}$ & 21.408965 & 10.217615 & 0.050000 \\
\hline $\mathrm{H}$ & 21.409437 & 5.210551 & 0.050000 \\
\hline $\mathrm{H}$ & 23.866661 & 10.217681 & 0.050000 \\
\hline $\mathrm{H}$ & 23.866985 & 5.211198 & 0.050000 \\
\hline $\mathrm{H}$ & 26.324131 & 10.217463 & 0.050000 \\
\hline $\mathrm{H}$ & 26.323476 & 5.211014 & 0.050000 \\
\hline $\mathrm{H}$ & 28.781339 & 10.217006 & 0.050000 \\
\hline $\mathrm{H}$ & 28.780360 & 5.210382 & 0.050000 \\
\hline $\mathrm{H}$ & 31.238466 & 10.216690 & 0.050000 \\
\hline $\mathrm{H}$ & 31.237411 & 5.209477 & 0.050000 \\
\hline $\mathrm{H}$ & 33.696582 & 10.216669 & 0.050000 \\
\hline $\mathrm{H}$ & 33.696140 & 5.208723 & 0.050000 \\
\hline $\mathrm{H}$ & 36.156456 & 10.216743 & 0.050000 \\
\hline $\mathrm{H}$ & 36.156910 & 5.208628 & 0.050000 \\
\hline $\mathrm{H}$ & 38.618400 & 10.216638 & 0.050000 \\
\hline $\mathrm{H}$ & 38.619624 & 5.209543 & 0.050000 \\
\hline $\mathrm{H}$ & 41.083639 & 10.216186 & 0.050000 \\
\hline $\mathrm{H}$ & 41.085639 & 5.211926 & 0.050000 \\
\hline $\mathrm{H}$ & 43.553946 & 10.217106 & 0.050000 \\
\hline $\mathrm{H}$ & 45.692927 & 8.966856 & 0.050000 \\
\hline $\mathrm{H}$ & 45.694322 & 6.467659 & 0.050000 \\
\hline & 43.557534 & 5.214118 & 0.050000 \\
\hline \multicolumn{4}{|c|}{ ene } \\
\hline C & 9.842067 & 5.600107 & 0.053000 \\
\hline
\end{tabular}




\begin{tabular}{|c|c|c|c|}
\hline C & 46.753859 & 5.600962 & 0.053000 \\
\hline & 12.315927 & 5.601594 & 0.053000 \\
\hline C & 14.779812 & 5.601335 & 0.053000 \\
\hline C & 17.239804 & 5.601309 & 0.053000 \\
\hline C & 41.815446 & 5.601646 & 0.053000 \\
\hline C & 39.355023 & 5.601639 & 0.053000 \\
\hline C & 44.279917 & 5.602429 & 0.053000 \\
\hline C & 19.698150 & 5.602136 & 0.053000 \\
\hline C & 36.896945 & 5.602211 & 0.053000 \\
\hline & 34.439813 & 5.602298 & 0.053000 \\
\hline & 31.982844 & 5.602437 & 0.053000 \\
\hline & 22.155499 & 5.603183 & 0.053000 \\
\hline C & 29.526023 & 5.603198 & 0.053000 \\
\hline & 24.612378 & 5.603838 & 0.053000 \\
\hline & 27.069286 & 5.603775 & 0.053000 \\
\hline & 33.214002 & 6.281112 & 0.053000 \\
\hline & 30.755744 & 6.281541 & 0.053000 \\
\hline & 35.673294 & 6.281311 & 0.053000 \\
\hline & 28.297962 & 6.282297 & 0.053000 \\
\hline & 18.461414 & 6.281119 & 0.053000 \\
\hline & 20.921462 & 6.281644 & 0.053000 \\
\hline C & 25.839854 & 6.282604 & 0.053000 \\
\hline C & 23.381099 & 6.282417 & 0.053000 \\
\hline C & 38.133265 & 6.281501 & 0.053000 \\
\hline C & 16.002628 & 6.281724 & 0.053000 \\
\hline & 40.592125 & 6.281892 & 0.053000 \\
\hline & 13.546766 & 6.282805 & 0.053000 \\
\hline C & 43.048583 & 6.283167 & 0.053000 \\
\hline & 11.095254 & 6.284075 & 0.053000 \\
\hline & 45.500910 & 6.285025 & 0.053000 \\
\hline & 8.657596 & 6.296062 & 0.053000 \\
\hline & 47.938580 & 6.296916 & 0.053000 \\
\hline 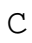 & 8.656807 & 7.721706 & 0.053000 \\
\hline C & 47.939088 & 7.722351 & 0.053000 \\
\hline C & 11.094520 & 7.736237 & 0.053000 \\
\hline C & 45.501391 & 7.737182 & 0.053000 \\
\hline$\gamma$ & 13.546164 & 7.740669 & 0.053000 \\
\hline 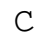 & 43.049030 & 7.741056 & 0.053000 \\
\hline C & 16.002277 & 7.743543 & 0.053000 \\
\hline C & 40.592481 & 7.743824 & 0.053000 \\
\hline C & 18.461178 & 7.744784 & 0.053000 \\
\hline C & 33.214073 & 7.744269 & 0.053000 \\
\hline C & 30.755840 & 7.743977 & 0.053000 \\
\hline C & 38.133451 & 7.745154 & 0.053000 \\
\hline C & 35.673362 & 7.745025 & 0.053000 \\
\hline C & 20.921289 & 7.745477 & 0.053000 \\
\hline $\mathrm{C}$ & 28.298100 & 7.744451 & 0.053000 \\
\hline C & 25.839908 & 7.745135 & 0.053000 \\
\hline C & 23.381018 & 7.745677 & 0.053000 \\
\hline $\mathrm{C}$ & 9.840719 & 8.418671 & 0.053000 \\
\hline C & 46.755199 & 8.419524 & 0.053000 \\
\hline C & 12.314418 & 8.420142 & 0.053000 \\
\hline C & 44.280867 & 8.420680 & 0.053000 \\
\hline C & 29.526457 & 8.422796 & 0.053000 \\
\hline C & 31.982914 & 8.422729 & 0.053000 \\
\hline C & 27.069581 & 8.423220 & 0.053000 \\
\hline . & 34.439649 & 8.423372 & 0.053000 \\
\hline
\end{tabular}




\begin{tabular}{|c|c|c|c|}
\hline C & 41.816202 & 8.423082 & 0.053000 \\
\hline C & 14.778885 & 8.422947 & 0.053000 \\
\hline C & 24.612417 & 8.423945 & 0.053000 \\
\hline C & 22.155091 & 8.424172 & 0.053000 \\
\hline C & 17.239351 & 8.424024 & 0.053000 \\
\hline C & 19.697652 & 8.424141 & 0.053000 \\
\hline C & 36.897030 & 8.424144 & 0.053000 \\
\hline C & 39.355561 & 8.424403 & 0.053000 \\
\hline $\mathrm{H}$ & 7.707130 & 8.258447 & 0.053000 \\
\hline $\mathrm{H}$ & 7.708386 & 5.758351 & 0.053000 \\
\hline $\mathrm{H}$ & 9.843461 & 9.511226 & 0.053000 \\
\hline $\mathrm{H}$ & 9.846141 & 4.507556 & 0.053000 \\
\hline $\mathrm{H}$ & 12.313723 & 9.513638 & 0.053000 \\
\hline $\mathrm{H}$ & 12.316776 & 4.508157 & 0.053000 \\
\hline $\mathrm{H}$ & 14.778741 & 9.516239 & 0.053000 \\
\hline $\mathrm{H}$ & 14.780249 & 4.508062 & 0.053000 \\
\hline $\mathrm{H}$ & 17.240127 & 9.517255 & 0.053000 \\
\hline $\mathrm{H}$ & 17.240501 & 4.508075 & 0.053000 \\
\hline $\mathrm{H}$ & 19.699068 & 9.517422 & 0.053000 \\
\hline $\mathrm{H}$ & 19.699981 & 4.508801 & 0.053000 \\
\hline $\mathrm{H}$ & 22.156930 & 9.517479 & 0.053000 \\
\hline $\mathrm{H}$ & 22.157948 & 4.509823 & 0.053000 \\
\hline $\mathrm{H}$ & 24.614175 & 9.517264 & 0.053000 \\
\hline $\mathrm{H}$ & 24.614146 & 4.510487 & 0.053000 \\
\hline $\mathrm{H}$ & 27.070437 & 9.516509 & 0.053000 \\
\hline $\mathrm{H}$ & 27.069742 & 4.510492 & 0.053000 \\
\hline $\mathrm{H}$ & 29.525984 & 9.516083 & 0.053000 \\
\hline $\mathrm{H}$ & 29.524824 & 4.509917 & 0.053000 \\
\hline $\mathrm{H}$ & 31.981030 & 9.516039 & 0.053000 \\
\hline $\mathrm{H}$ & 31.980907 & 4.509130 & 0.053000 \\
\hline $\mathrm{H}$ & 34.437092 & 9.516697 & 0.053000 \\
\hline $\mathrm{H}$ & 34.437889 & 4.508961 & 0.053000 \\
\hline $\mathrm{H}$ & 36.895350 & 9.517447 & 0.053000 \\
\hline $\mathrm{H}$ & 36.895249 & 4.508914 & 0.053000 \\
\hline $\mathrm{H}$ & 39.355314 & 9.517614 & 0.053000 \\
\hline $\mathrm{H}$ & 39.354033 & 4.508410 & 0.053000 \\
\hline $\mathrm{H}$ & 41.816617 & 9.516330 & 0.053000 \\
\hline $\mathrm{H}$ & 41.815188 & 4.508323 & 0.053000 \\
\hline $\mathrm{H}$ & 44.281150 & 9.514145 & 0.053000 \\
\hline $\mathrm{H}$ & 44.279761 & 4.508966 & 0.053000 \\
\hline $\mathrm{H}$ & 46.752419 & 9.512089 & 0.053000 \\
\hline $\mathrm{H}$ & 46.749621 & 4.508454 & 0.053000 \\
\hline $\mathrm{H}$ & 48.888762 & 8.259183 & 0.053000 \\
\hline $\mathrm{H}$ & 48.887746 & 5.759289 & 0.053000 \\
\hline \multicolumn{4}{|c|}{ 20] acene } \\
\hline C & 53.053457 & 5.970899 & 0.058217 \\
\hline C & 6.241640 & 5.971231 & 0.058893 \\
\hline C & 48.108122 & 5.973133 & 0.060752 \\
\hline C & 45.644043 & 5.973231 & 0.059203 \\
\hline $\mathrm{C}$ & 50.576102 & 5.973374 & 0.060941 \\
\hline C & 8.717680 & 5.973709 & 0.060378 \\
\hline C & 43.181678 & 5.973913 & 0.059239 \\
\hline C & 11.184193 & 5.974296 & 0.059322 \\
\hline C & 25.953646 & 5.974154 & 0.058083 \\
\hline C & 28.415202 & 5.974129 & 0.059565 \\
\hline $\mathrm{C}$ & 40.719832 & 5.974916 & 0.060157 \\
\hline C & 23.491536 & 5.974791 & 0.057235 \\
\hline
\end{tabular}




\begin{tabular}{|c|c|c|c|}
\hline C & 30.876148 & 5.974738 & 0.060975 \\
\hline & 13.646836 & 5.975572 & 0.058128 \\
\hline & 38.258655 & 5.975741 & 0.061597 \\
\hline C & 21.029336 & 5.975679 & 0.057778 \\
\hline & 33.337015 & 5.975557 & 0.061567 \\
\hline & 35.797682 & 5.976088 & 0.061870 \\
\hline & 18.568026 & 5.976514 & 0.058856 \\
\hline & 16.107547 & 5.976613 & 0.058561 \\
\hline & 44.420251 & 6.651961 & 0.059410 \\
\hline & 41.955264 & 6.652225 & 0.060303 \\
\hline & 27.185443 & 6.652478 & 0.059059 \\
\hline & 24.724045 & 6.652472 & 0.057619 \\
\hline & 22.261184 & 6.652962 & 0.057472 \\
\hline & 46.883584 & 6.652575 & 0.060195 \\
\hline & 29.645640 & 6.653048 & 0.060647 \\
\hline & 39.490645 & 6.653311 & 0.061355 \\
\hline & 32.105640 & 6.653738 & 0.061630 \\
\hline & 19.797418 & 6.653933 & 0.058387 \\
\hline & 49.343791 & 6.653742 & 0.061087 \\
\hline & 37.027569 & 6.654174 & 0.062526 \\
\hline & 34.566037 & 6.654401 & 0.061911 \\
\hline & 51.799394 & 6.654734 & 0.059550 \\
\hline & 17.333447 & 6.654942 & 0.058957 \\
\hline & 9.949285 & 6.654742 & 0.059798 \\
\hline & 12.408001 & 6.654714 & 0.058533 \\
\hline & 14.869941 & 6.655081 & 0.058199 \\
\hline & 7.495189 & 6.655287 & 0.059655 \\
\hline & 54.239197 & 6.666167 & 0.055847 \\
\hline & 5.056233 & 6.666626 & 0.056582 \\
\hline & 54.240203 & 8.091205 & 0.054655 \\
\hline & 5.055112 & 8.091808 & 0.055194 \\
\hline & 51.800301 & 8.106987 & 0.058474 \\
\hline & 7.494193 & 8.107474 & 0.058696 \\
\hline & 49.344602 & 8.111569 & 0.060141 \\
\hline & 9.948394 & 8.112518 & 0.059460 \\
\hline & 46.884098 & 8.114460 & 0.059850 \\
\hline & 27.185452 & 8.114222 & 0.058953 \\
\hline & 29.645522 & 8.114664 & 0.060835 \\
\hline & 24.724254 & 8.114690 & 0.057065 \\
\hline & 44.420621 & 8.115785 & 0.059640 \\
\hline & 32.105314 & 8.115477 & 0.061543 \\
\hline & 22.261681 & 8.115844 & 0.056692 \\
\hline & 12.407363 & 8.116466 & 0.058789 \\
\hline 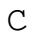 & 41.955684 & 8.116397 & 0.060435 \\
\hline & 34.565848 & 8.116593 & 0.061464 \\
\hline & 39.490903 & 8.117008 & 0.061233 \\
\hline & 37.027585 & 8.117120 & 0.061399 \\
\hline & 19.797828 & 8.117669 & 0.057658 \\
\hline & 14.869602 & 8.118903 & 0.058729 \\
\hline C & 17.333591 & 8.119252 & 0.058671 \\
\hline C & 53.055682 & 8.788355 & 0.056225 \\
\hline & 6.239417 & 8.788985 & 0.056568 \\
\hline & 50.577921 & 8.790012 & 0.058895 \\
\hline & 8.715574 & 8.791036 & 0.059057 \\
\hline & 25.953925 & 8.792506 & 0.057346 \\
\hline$C$ & 28.414864 & 8.792794 & 0.059731 \\
\hline & 23.492326 & 8.792973 & 0.056075 \\
\hline
\end{tabular}




\begin{tabular}{|c|c|c|c|}
\hline $\mathrm{C}$ & 48.109227 & 8.792884 & 0.059429 \\
\hline $\mathrm{C}$ & 30.875588 & 8.793584 & 0.061310 \\
\hline C & 33.336327 & 8.794301 & 0.061015 \\
\hline $\mathrm{C}$ & 45.644740 & 8.793928 & 0.059108 \\
\hline C & 21.030563 & 8.794231 & 0.056264 \\
\hline $\mathrm{C}$ & 43.182340 & 8.794077 & 0.059681 \\
\hline C & 40.720632 & 8.794278 & 0.060256 \\
\hline C & 35.797541 & 8.794871 & 0.060605 \\
\hline C & 38.259092 & 8.794824 & 0.060741 \\
\hline C & 11.182418 & 8.794769 & 0.059375 \\
\hline C & 18.569009 & 8.796216 & 0.058040 \\
\hline $\mathrm{C}$ & 13.645654 & 8.796874 & 0.059002 \\
\hline C & 16.107420 & 8.797379 & 0.059078 \\
\hline $\mathrm{H}$ & 4.105488 & 8.628998 & 0.052760 \\
\hline $\mathrm{H}$ & 4.107255 & 6.128326 & 0.055353 \\
\hline $\mathrm{H}$ & 6.239858 & 9.881544 & 0.055431 \\
\hline $\mathrm{H}$ & 6.243800 & 4.878711 & 0.059628 \\
\hline $\mathrm{H}$ & 8.714926 & 9.884517 & 0.058508 \\
\hline $\mathrm{H}$ & 8.718758 & 4.880200 & 0.060534 \\
\hline $\mathrm{H}$ & 11.181196 & 9.888168 & 0.059699 \\
\hline $\mathrm{H}$ & 11.184603 & 4.880907 & 0.059402 \\
\hline $\mathrm{H}$ & 13.643846 & 9.890311 & 0.059975 \\
\hline $\mathrm{H}$ & 13.646114 & 4.882178 & 0.058410 \\
\hline $\mathrm{H}$ & 16.106613 & 9.890866 & 0.059710 \\
\hline $\mathrm{H}$ & 16.106527 & 4.883150 & 0.058667 \\
\hline $\mathrm{H}$ & 18.570255 & 9.889691 & 0.057500 \\
\hline $\mathrm{H}$ & 18.567470 & 4.883037 & 0.058998 \\
\hline $\mathrm{H}$ & 21.032952 & 9.887697 & 0.054519 \\
\hline $\mathrm{H}$ & 21.030345 & 4.882208 & 0.057533 \\
\hline $\mathrm{H}$ & 23.494877 & 9.886403 & 0.054958 \\
\hline $\mathrm{H}$ & 23.493310 & 4.881337 & 0.056593 \\
\hline $\mathrm{H}$ & 25.955859 & 9.885933 & 0.056090 \\
\hline $\mathrm{H}$ & 25.955227 & 4.880733 & 0.057052 \\
\hline $\mathrm{H}$ & 28.415126 & 9.886187 & 0.058965 \\
\hline $\mathrm{H}$ & 28.416063 & 4.880741 & 0.058344 \\
\hline $\mathrm{H}$ & 30.874595 & 9.886984 & 0.060693 \\
\hline $\mathrm{H}$ & 30.875825 & 4.881360 & 0.059959 \\
\hline $\mathrm{H}$ & 33.334347 & 9.887723 & 0.059750 \\
\hline $\mathrm{H}$ & 33.335736 & 4.882157 & 0.060803 \\
\hline $\mathrm{H}$ & 35.795509 & 9.888286 & 0.058820 \\
\hline $\mathrm{H}$ & 35.795495 & 4.882650 & 0.061261 \\
\hline $\mathrm{H}$ & 38.257512 & 9.888287 & 0.059533 \\
\hline $\mathrm{H}$ & 38.256425 & 4.882240 & 0.060620 \\
\hline $\mathrm{H}$ & 40.719814 & 9.887776 & 0.058550 \\
\hline $\mathrm{H}$ & 40.718316 & 4.881401 & 0.058436 \\
\hline $\mathrm{H}$ & 43.181905 & 9.887557 & 0.058586 \\
\hline $\mathrm{H}$ & 43.180881 & 4.880429 & 0.057744 \\
\hline $\mathrm{H}$ & 45.644506 & 9.887349 & 0.057818 \\
\hline $\mathrm{H}$ & 45.643744 & 4.879833 & 0.057578 \\
\hline $\mathrm{H}$ & 48.109288 & 9.886333 & 0.057506 \\
\hline $\mathrm{H}$ & 48.107640 & 4.879727 & 0.059747 \\
\hline $\mathrm{H}$ & 50.579359 & 9.883585 & 0.057553 \\
\hline $\mathrm{H}$ & 50.575708 & 4.879866 & 0.061228 \\
\hline $\mathrm{H}$ & 53.056819 & 9.880959 & 0.055068 \\
\hline $\mathrm{H}$ & 55.190246 & 8.627865 & 0.052099 \\
\hline $\mathrm{H}$ & 55.188165 & 6.127833 & 0.053909 \\
\hline $\mathrm{H}$ & 53.052355 & 4.878375 & 0.058696 \\
\hline
\end{tabular}


[40] acene

\begin{tabular}{|c|c|c|c|}
\hline $\mathrm{C}$ & 39.959859 & 6.086456 & 5.000000 \\
\hline $\mathrm{C}$ & 66.800126 & 6.086456 & 5.000000 \\
\hline $\mathrm{C}$ & 42.399688 & 6.086442 & 5.000000 \\
\hline $\mathrm{C}$ & 64.360297 & 6.086442 & 5.000000 \\
\hline $\mathrm{C}$ & 37.519703 & 6.086482 & 5.000000 \\
\hline $\mathrm{C}$ & 69.240271 & 6.086482 & 5.000000 \\
\hline $\mathrm{C}$ & 32.638456 & 6.086552 & 5.000000 \\
\hline $\mathrm{C}$ & 74.121529 & 6.086552 & 5.000000 \\
\hline $\mathrm{C}$ & 47.279828 & 6.086538 & 5.000000 \\
\hline $\mathrm{C}$ & 59.480168 & 6.086538 & 5.000000 \\
\hline $\mathrm{C}$ & 35.079300 & 6.086745 & 5.000000 \\
\hline $\mathrm{C}$ & 71.680685 & 6.086745 & 5.000000 \\
\hline $\mathrm{C}$ & 44.839796 & 6.086720 & 5.000000 \\
\hline $\mathrm{C}$ & 61.920189 & 6.086720 & 5.000000 \\
\hline $\mathrm{C}$ & 49.719894 & 6.086563 & 5.000000 \\
\hline $\mathrm{C}$ & 57.040079 & 6.086563 & 5.000000 \\
\hline $\mathrm{C}$ & 52.159887 & 6.086668 & 5.000000 \\
\hline $\mathrm{C}$ & 54.600098 & 6.086668 & 5.000000 \\
\hline $\mathrm{C}$ & 30.197338 & 6.086857 & 5.000000 \\
\hline $\mathrm{C}$ & 76.562647 & 6.086857 & 5.000000 \\
\hline $\mathrm{C}$ & 27.755304 & 6.087131 & 5.000000 \\
\hline $\mathrm{C}$ & 79.004681 & 6.087131 & 5.000000 \\
\hline $\mathrm{C}$ & 15.527468 & 6.087986 & 5.000000 \\
\hline $\mathrm{C}$ & 91.232517 & 6.087986 & 5.000000 \\
\hline $\mathrm{C}$ & 22.868608 & 6.087608 & 5.000000 \\
\hline $\mathrm{C}$ & 83.891377 & 6.087608 & 5.000000 \\
\hline $\mathrm{C}$ & 20.423543 & 6.087819 & 5.000000 \\
\hline $\mathrm{C}$ & 86.336431 & 6.087819 & 5.000000 \\
\hline $\mathrm{C}$ & 25.312431 & 6.087584 & 5.000000 \\
\hline $\mathrm{C}$ & 81.447543 & 6.087584 & 5.000000 \\
\hline $\mathrm{C}$ & 17.976630 & 6.087944 & 5.000000 \\
\hline $\mathrm{C}$ & 88.783355 & 6.087944 & 5.000000 \\
\hline $\mathrm{C}$ & 13.075076 & 6.088174 & 5.000000 \\
\hline $\mathrm{C}$ & 93.684909 & 6.088174 & 5.000000 \\
\hline $\mathrm{C}$ & 10.618312 & 6.088933 & 5.000000 \\
\hline $\mathrm{C}$ & 96.141685 & 6.088933 & 5.000000 \\
\hline $\mathrm{C}$ & 5.682657 & 6.090871 & 5.000000 \\
\hline $\mathrm{C}$ & 101.077328 & 6.090871 & 5.000000 \\
\hline $\mathrm{C}$ & 8.155857 & 6.090977 & 5.000000 \\
\hline $\mathrm{C}$ & 98.604128 & 6.090977 & 5.000000 \\
\hline $\mathrm{C}$ & 16.747832 & 6.768305 & 5.000000 \\
\hline $\mathrm{C}$ & 90.012153 & 6.768305 & 5.000000 \\
\hline $\mathrm{C}$ & 19.198644 & 6.768618 & 5.000000 \\
\hline $\mathrm{C}$ & 87.561341 & 6.768618 & 5.000000 \\
\hline $\mathrm{C}$ & 21.646547 & 6.769014 & 5.000000 \\
\hline $\mathrm{C}$ & 85.113438 & 6.769014 & 5.000000 \\
\hline $\mathrm{C}$ & 14.294171 & 6.768434 & 5.000000 \\
\hline $\mathrm{C}$ & 92.465814 & 6.768434 & 5.000000 \\
\hline $\mathrm{C}$ & 24.091652 & 6.769267 & 5.000000 \\
\hline $\mathrm{C}$ & 82.668333 & 6.769267 & 5.000000 \\
\hline $\mathrm{C}$ & 53.379998 & 6.769278 & 5.000000 \\
\hline $\mathrm{C}$ & 43.619514 & 6.769205 & 5.000000 \\
\hline $\mathrm{C}$ & 63.140471 & 6.769205 & 5.000000 \\
\hline $\mathrm{C}$ & 26.535443 & 6.769545 & 5.000000 \\
\hline $\mathrm{C}$ & 80.224542 & 6.769545 & 5.000000 \\
\hline $\mathrm{C}$ & 48.499900 & 6.769407 & 5.000000 \\
\hline
\end{tabular}




\begin{tabular}{|c|c|c|c|}
\hline 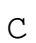 & 58.260085 & 6.769407 & 5.000000 \\
\hline & 50.939734 & 6.769540 & 5.000000 \\
\hline C & 55.820251 & 6.769540 & 5.000000 \\
\hline C & 28.977353 & 6.769499 & 5.000000 \\
\hline $\mathrm{C}$ & 77.782632 & 6.769499 & 5.000000 \\
\hline & 33.858644 & 6.769273 & 5.000000 \\
\hline C & 72.901341 & 6.769273 & 5.000000 \\
\hline & 46.060043 & 6.769498 & 5.000000 \\
\hline & 60.699931 & 6.769498 & 5.000000 \\
\hline & 41.179417 & 6.769425 & 5.000000 \\
\hline & 65.580580 & 6.769425 & 5.000000 \\
\hline & 38.739527 & 6.769347 & 5.000000 \\
\hline & 68.020458 & 6.769347 & 5.000000 \\
\hline & 11.839864 & 6.769285 & 5.000000 \\
\hline & 94.920121 & 6.769285 & 5.000000 \\
\hline & 31.418277 & 6.769522 & 5.000000 \\
\hline & 75.341708 & 6.769522 & 5.000000 \\
\hline & 36.299513 & 6.769493 & 5.000000 \\
\hline & 70.460472 & 6.769493 & 5.000000 \\
\hline & 9.386440 & 6.771104 & 5.000000 \\
\hline & 97.373545 & 6.771104 & 5.000000 \\
\hline & 6.935310 & 6.774280 & 5.000000 \\
\hline & 99.824675 & 6.774280 & 5.000000 \\
\hline & 4.498298 & 6.787378 & 5.000000 \\
\hline & 102.261687 & 6.787378 & 5.000000 \\
\hline & 4.498298 & 8.212622 & 5.000000 \\
\hline C & 102.261687 & 8.212622 & 5.000000 \\
\hline & 6.935310 & 8.225720 & 5.000000 \\
\hline & 99.824675 & 8.225720 & 5.000000 \\
\hline & 9.386440 & 8.228896 & 5.000000 \\
\hline & 97.373545 & 8.228896 & 5.000000 \\
\hline & 36.299513 & 8.230507 & 5.000000 \\
\hline & 70.460472 & 8.230507 & 5.000000 \\
\hline & 31.418277 & 8.230478 & 5.000000 \\
\hline & 75.341708 & 8.230478 & 5.000000 \\
\hline & 11.839864 & 8.230715 & 5.000000 \\
\hline & 94.920121 & 8.230715 & 5.000000 \\
\hline & 38.739527 & 8.230653 & 5.000000 \\
\hline $\mathrm{C}$ & 68.020458 & 8.230653 & 5.000000 \\
\hline & 41.179417 & 8.230575 & 5.000000 \\
\hline & 65.580580 & 8.230575 & 5.000000 \\
\hline & 46.060043 & 8.230502 & 5.000000 \\
\hline & 60.699931 & 8.230502 & 5.000000 \\
\hline C & 33.858644 & 8.230727 & 5.000000 \\
\hline C & 72.901341 & 8.230727 & 5.000000 \\
\hline $\mathrm{C}$ & 28.977353 & 8.230501 & 5.000000 \\
\hline C & 77.782632 & 8.230501 & 5.000000 \\
\hline & 50.939734 & 8.230460 & 5.000000 \\
\hline & 55.820251 & 8.230460 & 5.000000 \\
\hline $\mathrm{C}$ & 48.499900 & 8.230593 & 5.000000 \\
\hline C & 58.260085 & 8.230593 & 5.000000 \\
\hline C & 26.535443 & 8.230454 & 5.000000 \\
\hline $\mathrm{C}$ & 80.224542 & 8.230454 & 5.000000 \\
\hline & 43.619514 & 8.230795 & 5.000000 \\
\hline & 63.140471 & 8.230795 & 5.000000 \\
\hline & 53.379998 & 8.230722 & 5.000000 \\
\hline & 24.091652 & 8.230733 & 5.000000 \\
\hline
\end{tabular}




\begin{tabular}{|c|c|c|c|}
\hline E & 82.668333 & 8.230733 & 5.000000 \\
\hline C & 14.294171 & 8.231566 & 5.000000 \\
\hline . & 92.465814 & 8.231566 & 5.000000 \\
\hline & 21.646547 & 8.230984 & 5.000000 \\
\hline C & 85.113438 & 8.230984 & 5.000000 \\
\hline & 19.198644 & 8.231382 & 5.000000 \\
\hline $\mathrm{C}$ & 87.561341 & 8.231382 & 5.000000 \\
\hline & 16.747832 & 8.231695 & 5.000000 \\
\hline & 90.012153 & 8.231695 & 5.000000 \\
\hline & 8.155857 & 8.909021 & 5.000000 \\
\hline & 98.604128 & 8.909021 & 5.000000 \\
\hline & 5.682657 & 8.909129 & 5.000000 \\
\hline & 101.077328 & 8.909129 & 5.000000 \\
\hline & 10.618312 & 8.911067 & 5.000000 \\
\hline & 96.141685 & 8.911067 & 5.000000 \\
\hline & 13.075076 & 8.911826 & 5.000000 \\
\hline & 93.684909 & 8.911826 & 5.000000 \\
\hline & 17.976630 & 8.912056 & 5.000000 \\
\hline C & 88.783355 & 8.912056 & 5.000000 \\
\hline C & 25.312431 & 8.912416 & 5.000000 \\
\hline C & 81.447543 & 8.912416 & 5.000000 \\
\hline C & 20.423543 & 8.912181 & 5.000000 \\
\hline C & 86.336431 & 8.912181 & 5.000000 \\
\hline & 22.868608 & 8.912392 & 5.000000 \\
\hline$C$ & 83.891377 & 8.912392 & 5.000000 \\
\hline & 15.527468 & 8.912013 & 5.000000 \\
\hline & 91.232517 & 8.912013 & 5.000000 \\
\hline & 27.755304 & 8.912871 & 5.000000 \\
\hline C & 79.004681 & 8.912871 & 5.000000 \\
\hline 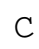 & 30.197338 & 8.913143 & 5.000000 \\
\hline $\mathrm{C}$ & 76.562647 & 8.913143 & 5.000000 \\
\hline & 52.159887 & 8.913332 & 5.000000 \\
\hline & 54.600098 & 8.913332 & 5.000000 \\
\hline C & 49.719894 & 8.913437 & 5.000000 \\
\hline z & 57.040079 & 8.913437 & 5.000000 \\
\hline C & 44.839796 & 8.913279 & 5.000000 \\
\hline $\mathrm{C}$ & 61.920189 & 8.913279 & 5.000000 \\
\hline & 35.079300 & 8.913255 & 5.000000 \\
\hline & 71.680685 & 8.913255 & 5.000000 \\
\hline C & 47.279828 & 8.913462 & 5.000000 \\
\hline C & 59.480168 & 8.913462 & 5.000000 \\
\hline C & 32.638456 & 8.913448 & 5.000000 \\
\hline & 74.121529 & 8.913448 & 5.000000 \\
\hline C & 37.519703 & 8.913518 & 5.000000 \\
\hline $\mathrm{C}$ & 69.240271 & 8.913518 & 5.000000 \\
\hline $\mathrm{C}$ & 42.399688 & 8.913558 & 5.000000 \\
\hline $\mathrm{C}$ & 64.360297 & 8.913558 & 5.000000 \\
\hline C & 39.959859 & 8.913544 & 5.000000 \\
\hline C & 66.800126 & 8.913544 & 5.000000 \\
\hline $\mathrm{H}$ & 3.549139 & 8.749956 & 5.000000 \\
\hline $\mathrm{H}$ & 3.549139 & 6.250044 & 5.000000 \\
\hline $\mathrm{H}$ & 5.688673 & 10.001497 & 5.000000 \\
\hline $\mathrm{H}$ & 5.688673 & 4.998503 & 5.000000 \\
\hline $\mathrm{H}$ & 8.157481 & 10.002379 & 5.000000 \\
\hline & 8.157481 & 4.997621 & 5.000000 \\
\hline & 10.620516 & 10.004292 & 5.000000 \\
\hline & 10.620516 & 4.995708 & 5.000000 \\
\hline
\end{tabular}




\begin{tabular}{|c|c|c|c|}
\hline $\mathrm{H}$ & 13.078485 & 10.005059 & 5.000000 \\
\hline $\mathrm{H}$ & 13.078485 & 4.994941 & 5.000000 \\
\hline $\mathrm{H}$ & 15.531555 & 10.005308 & 5.000000 \\
\hline $\mathrm{H}$ & 15.531555 & 4.994692 & 5.000000 \\
\hline $\mathrm{H}$ & 17.980535 & 10.005288 & 5.000000 \\
\hline $\mathrm{H}$ & 17.980535 & 4.994712 & 5.000000 \\
\hline $\mathrm{H}$ & 20.427062 & 10.005365 & 5.000000 \\
\hline $\mathrm{H}$ & 20.427062 & 4.994634 & 5.000000 \\
\hline $\mathrm{H}$ & 22.871337 & 10.005549 & 5.000000 \\
\hline $\mathrm{H}$ & 22.871337 & 4.994451 & 5.000000 \\
\hline $\mathrm{H}$ & 25.314283 & 10.005499 & 5.000000 \\
\hline $\mathrm{H}$ & 25.314283 & 4.994501 & 5.000000 \\
\hline $\mathrm{H}$ & 27.756234 & 10.005949 & 5.000000 \\
\hline $\mathrm{H}$ & 27.756234 & 4.994051 & 5.000000 \\
\hline $\mathrm{H}$ & 30.197757 & 10.006205 & 5.000000 \\
\hline $\mathrm{H}$ & 30.197757 & 4.993795 & 5.000000 \\
\hline $\mathrm{H}$ & 32.638529 & 10.006507 & 5.000000 \\
\hline $\mathrm{H}$ & 32.638529 & 4.993493 & 5.000000 \\
\hline $\mathrm{H}$ & 35.079442 & 10.006331 & 5.000000 \\
\hline $\mathrm{H}$ & 35.079442 & 4.993669 & 5.000000 \\
\hline $\mathrm{H}$ & 37.519733 & 10.006565 & 5.000000 \\
\hline $\mathrm{H}$ & 37.519733 & 4.993435 & 5.000000 \\
\hline $\mathrm{H}$ & 39.960079 & 10.006603 & 5.000000 \\
\hline $\mathrm{H}$ & 39.960079 & 4.993397 & 5.000000 \\
\hline $\mathrm{H}$ & 42.399886 & 10.006592 & 5.000000 \\
\hline $\mathrm{H}$ & 42.399886 & 4.993408 & 5.000000 \\
\hline $\mathrm{H}$ & 44.840126 & 10.006360 & 5.000000 \\
\hline $\mathrm{H}$ & 44.840126 & 4.993640 & 5.000000 \\
\hline $\mathrm{H}$ & 47.279991 & 10.006482 & 5.000000 \\
\hline $\mathrm{H}$ & 47.279991 & 4.993518 & 5.000000 \\
\hline $\mathrm{H}$ & 49.720118 & 10.006446 & 5.000000 \\
\hline $\mathrm{H}$ & 49.720118 & 4.993554 & 5.000000 \\
\hline $\mathrm{H}$ & 52.159876 & 10.006356 & 5.000000 \\
\hline $\mathrm{H}$ & 52.159876 & 4.993644 & 5.000000 \\
\hline $\mathrm{H}$ & 54.600109 & 10.006356 & 5.000000 \\
\hline $\mathrm{H}$ & 54.600109 & 4.993644 & 5.000000 \\
\hline $\mathrm{H}$ & 57.039867 & 10.006446 & 5.000000 \\
\hline $\mathrm{H}$ & 57.039867 & 4.993554 & 5.000000 \\
\hline $\mathrm{H}$ & 59.479994 & 10.006482 & 5.000000 \\
\hline $\mathrm{H}$ & 59.479994 & 4.993518 & 5.000000 \\
\hline $\mathrm{H}$ & 61.919859 & 10.006360 & 5.000000 \\
\hline $\mathrm{H}$ & 61.919859 & 4.993640 & 5.000000 \\
\hline $\mathrm{H}$ & 64.360099 & 10.006592 & 5.000000 \\
\hline $\mathrm{H}$ & 64.360099 & 4.993408 & 5.000000 \\
\hline $\mathrm{H}$ & 66.799906 & 10.006603 & 5.000000 \\
\hline $\mathrm{H}$ & 66.799906 & 4.993397 & 5.000000 \\
\hline $\mathrm{H}$ & 69.240252 & 10.006565 & 5.000000 \\
\hline $\mathrm{H}$ & 69.240252 & 4.993435 & 5.000000 \\
\hline $\mathrm{H}$ & 71.680543 & 10.006331 & 5.000000 \\
\hline $\mathrm{H}$ & 71.680543 & 4.993669 & 5.000000 \\
\hline $\mathrm{H}$ & 74.121456 & 10.006507 & 5.000000 \\
\hline $\mathrm{H}$ & 74.121456 & 4.993493 & 5.000000 \\
\hline $\mathrm{H}$ & 76.562228 & 10.006205 & 5.00000 \\
\hline $\mathrm{H}$ & 76.562228 & 4.993795 & 5.00000 \\
\hline $\mathrm{H}$ & 79.003751 & 10.005949 & 5.000000 \\
\hline $\mathrm{H}$ & 79.003751 & 4.994051 & 5.000000 \\
\hline $\mathrm{H}$ & 81.445702 & 10.005499 & 5.00000 \\
\hline
\end{tabular}




$\begin{array}{rrrr}\mathrm{H} & 81.445702 & 4.994501 & 5.000000 \\ \mathrm{H} & 83.888636 & 10.005549 & 5.000000 \\ \mathrm{H} & 83.888636 & 4.994451 & 5.000000 \\ \mathrm{H} & 86.332923 & 10.005365 & 5.000000 \\ \mathrm{H} & 86.332923 & 4.994634 & 5.000000 \\ \mathrm{H} & 88.779450 & 10.005288 & 5.000000 \\ \mathrm{H} & 88.779450 & 4.994712 & 5.000000 \\ \mathrm{H} & 91.228441 & 10.005308 & 5.000000 \\ \mathrm{H} & 91.228441 & 4.994692 & 5.000000 \\ \mathrm{H} & 93.681489 & 10.005059 & 5.000000 \\ \mathrm{H} & 93.681489 & 4.994941 & 5.000000 \\ \mathrm{H} & 96.139469 & 10.004292 & 5.000000 \\ \mathrm{H} & 96.139469 & 4.995708 & 5.000000 \\ \mathrm{H} & 98.602492 & 10.002379 & 5.000000 \\ \mathrm{H} & 98.602492 & 4.997621 & 5.000000 \\ \mathrm{H} & 101.071312 & 10.001497 & 5.000000 \\ \mathrm{H} & 101.071312 & 4.998503 & 5.000000 \\ \mathrm{H} & 103.210846 & 8.749956 & 5.000000 \\ \mathrm{H} & 103.210846 & 6.250044 & 5.000000\end{array}$

